\title{
Coronavirus Disease 2019 (COVID-19) And The Risk of Hypertensive Disorders of Pregnancy: A Retrospective Cohort Study
}

\author{
Ascension St. John Hospital \\ Fareeza Afzal \\ Ascension St. John Hospital \\ Susanna Szpunar \\ Ascension St. John Hospital \\ MaKenzie Tremp \\ Ascension Providence Hospital \\ Karlee Grace \\ Ascension Macomb-Oakland Hospital \\ Marina Liovas \\ St. George's University \\ Muhammad Aslam \\ Michigan State University
}

Michael Baracy ( $\square$ Michael.Baracy@ascension.org )

\section{Research Article}

Keywords: COVID-19, SARs-CoV-2, Pregnancy, Preeclampsia, Hypertensive disorders of pregnancy, Preterm birth

Posted Date: June 25th, 2021

DOI: https://doi.org/10.21203/rs.3.rs-583136/v1

License: (a) This work is licensed under a Creative Commons Attribution 4.0 International License. Read Full License 


\section{Abstract}

Purpose: To evaluate the association of hypertensive disorders of pregnancy with SARs-CoV-2 infection in pregnant women.

Methods: We conducted a retrospective cohort study of all pregnant patients with positive SARs-CoV-2 molecular test at four participating hospitals located in a large metropolitan city and who delivered between February $1^{\text {st }}$ and November $24^{\text {th }}, 2020$. Patients who tested positive during their pregnancy and delivered (index cases) were compared to the three subsequent deliveries of patients who tested negative (controls) at the same institution. We evaluated the impact of COVID-19 on the development of hypertensive disorders of pregnancy.

Results: A total of 280 patients were included in the study: 70 patients who tested positive for Coronavirus Disease-2019 and 210 matched controls. Compared with pregnancies negative for SARSCoV-2 infection, COVID-19 was associated with an increased risk of developing a hypertensive disorders of pregnancy (OR $3.68,95 \% \mathrm{Cl} 1.67-8.10)$. Overall rates of preeclampsia with severe features were significantly higher in patients with a COVID-19 diagnosis ( $18.6 \%$ vs $7.1 \%, p=0.006)$. Of COVID-19 positive patients, an early SARS-CoV-2 infection (prior to 32 week's gestation) conferred a higher risk of hypertensive disorders in pregnancy $(\mathrm{OR}=6.29, \mathrm{Cl} 1.64-24.07 ; \mathrm{p}=0.007)$. There was no difference in route of delivery, preterm birth, intrauterine growth restriction, intrauterine fetal demise, or pregnancy and fetal outcomes.

Conclusion: COVID-19 is a risk factor for hypertensive disorders of pregnancy.

\section{Tweetable Abstract}

Patients who test positive for COVID-19 during their pregnancy are at increased risk of developing a hypertensive disorder of pregnancy compared to pregnant patients who test negative for COVID-19. Earlier SARs-CoV-2 infection results in increased risk of developing a hypertensive disorder. Furthermore, even patients who are asymptomatic but positive for SARs-CoV-2 during their pregnancy have a higher risk of developing a hypertensive disorder of pregnancy.

\section{Introduction}

In December 2019, reports from China described a cluster of cases of pneumonia that were believed to be linked to the Huanan Seafood Wholesale Market in Wuhan, Hubei Province of China[1]. By early January of 2020, Chinese health authorities confirmed that the outbreak was caused by a novel coronavirus that differed from both severe acute respiratory syndrome coronavirus (SARS-CoV) and Middle East respiratory syndrome coronavirus (MERS-CoV)[2]. The virus would subsequently be named severe acute respiratory syndrome coronavirus-2 (SARS-CoV-2), the agent responsible for the human coronavirus disease-2019 (COVID-19). In March of 2021, COVID-19 cases surpassed 126 million globally and have been responsible for nearly 2.8 million deaths[3]. Of the 223 countries with confirmed cases, the United 
States and its territories has become the epicenter for COVID-19, accounting for approximately one-fourth of cases globally.

Early reports of COVID-19 in pregnancy suggested an association between SARS-CoV-2 infection and increased risk of hypertensive disorders of pregnancy (HDP), specifically gestational hypertension, preeclampsia, and preeclampsia with severe features. These reports, however, were mainly confined to case reports[4-6] or systematic reviews that incorporated multiple coronavirus species, namely SARS$\mathrm{CoV}$ and MERS-CoV in addition to SARS-CoV-2 [7-8]. Although there have more recent publications of pregnancy outcomes in patients diagnosed with COVID-19 [9-15], including systematic reviews[9, 1112], few reports have tested the association between SARS-CoV-2 infection and HDP. Furthermore, most systematic reviews have relied on large case series. In studies that have examined relationship between COVID-19 and HDP, outcomes were based on composite indices[16-17], were part of large cohort studies not powered for the outcome [9, 18-19], or lacked outcome data on a comparator group of individuals who tested negative for SARS-CoV-2 [20-22]. In addition, in systematic reviews without an appropriate control group, assumptions regarding associations between SARS-CoV-2 infections and hypertensive disorders of pregnancy are plagued by confounding and biases.

In this historical cohort study, we compared pregnant women who tested positive for SARS-CoV-2 within the course of their pregnancy to pregnant women who tested negative. The primary objective of this study was to compare the incidence of HDP between the two groups. We hypothesized that there would be higher rates of HDP in pregnancies complicated by COVID-19. Secondary outcomes included pregnancy outcomes, maternal complications, and neonatal outcomes.

\section{Methods}

This is a historical cohort study of all pregnant patients who tested positive for SARs-CoV-2 by molecular or polymerase chain reaction (PCR) based tests at four participating community hospitals located in a large metropolitan city and who delivered between February 1st and November 24th, 2020. Patients with positive antibody tests alone were excluded from the study. Index cases were defined as any patient who tested positive at any point during their pregnancy, regardless of symptoms, and who delivered withing the study period. Undelivered patients who tested positive for SARs-CoV-2 were not included. This study was approved by the Ascension St. John and Ascension Providence Institutional Review Boards (IRB) (Reference \#1617953) and granted exempt status with an approved waiver of informed consent. No funds were received for this work. All methods were carried out in accordance with relevant guidelines and regulations.

Index COVID-19 cases were compared to the subsequent three deliveries (controls) of patients who were asymptomatic or tested negative. If any of the three subsequent deliveries following an index case tested positive for SARs-CoV-2, that patient was treated as a new index case and the next negative case was used as a control. The subsequent three SARs-CoV-2 negative deliveries were then matched to the new index case. Ultimately, a 3:1 ratio of controls-to-index cases was achieved. 
Given the severe shortage of test kits in the initial stage of the study, only symptomatic patients and patients undergoing a cesarean delivery were tested for SARs-CoV-2. Starting in July, participating hospitals implemented universal SARS-CoV-2 testing for all patients admitted to the labor and delivery units. Thus, $32.1 \%$ of patients included in the control group were asymptomatic patients without suspicion for COVID-19.

Descriptive data were collected on index cases and controls. Data were abstracted from the electronic medical record (EMR) common to the four participating institutions. Abstracted data included patient demographics, medical histories and comorbid conditions, social history and recreational drug/substance use, gravidity, parity, obstetrical history, order of pregnancy (e.g. singleton v. higher order pregnancies), past pregnancy complications, current pregnancy complications, body mass index (BMI), COVID-19 self-reported symptoms, clinical signs of COVID-19, vital signs, laboratory values and inflammatory markers, imaging results, severity of disease, treatments, interventions and maternal and fetal outcomes. Race and ethnicity were self-reported at the time of admission. Gestational age (GA) was calculated based on the best obstetric estimate of the expected date of delivery (earliest ultrasound or sure last menstrual period). GA at COVID-19 diagnosis was determined by the date of the first positive SARs-CoV-2 test. Due to the limited imaging in the control group, analysis of imaging findings was omitted the from final analysis.

Maternal outcomes included gestational hypertension, preeclampsia without severe features, preeclampsia with severe features, eclampsia, hemolysis, elevated liver enzymes, and low platelets (HELLP) syndrome, gestational diabetes, intrauterine growth restriction, preterm labor, preterm birth, prelabor rupture of the membranes (PROM), preterm prelabor rupture of the membranes (PPROM), cervical insufficiency, venous thromboembolism, and placental abruption. HDP were define according to the American College of Obstetricians and Gynecologist criteria for gestational hypertension, preeclampsia with and without severe features, eclampsia, chronic hypertension with superimposed preeclampsia and HELLP syndrome. Neonatal outcomes were limited to the need for neonatal intensive care, neonatal demise, need for higher acuity care (i.e. special care nursery), or no intervention.

Data collection was coordinated by the principal investigator at each participating site. Data quality checks were done on a continuous basis, with reabstraction of data randomly assigned to cases across all sites. Demographic characteristics of index cases and controls were compared using descriptive statistics, including frequencies, mean and standard deviation for normally distributed continuous variables and median and interquartile range for nonnormal continuous data. Race and ethnicity were based on the National Institute of health $(\mathrm{NIH})$ categories. All patients who identified as Hispanic ethnic were grouped as "Hispanic." For analysis purposes, a "race reduced" or "all other" category included Native Hawaiian, Other Pacific Islander, American Indian or Alaskan Native, or self-reported other. These categories were combined to enable comparisons between groups, as these groups had lower frequencies of patients. 
Univariable analysis was conducted with Student's t-test, the chi-squared test, and analysis of variance (ANOVA). Multivariable analyses was done using logistic regression. From the univariable analysis, all variables associated with HDP or with COVID-19 status, with a p-value of 0.09 or less, were entered into a multivariable logistic regression equation using a forward likelihood ratio algorithm. The model with lowest - $2 \log$ likelihood value was taken to be the best model for the data. Logistic regression results are reported as the odds ratio (OR) and 95\% confidence interval. All data were analyzed using SPSS v. 27.0 and a $p$-value less than 0.05 was considered to indicate statistical significance.

\section{Results}

Our primary analysis included 5,962 patients who delivered at four participating hospitals during the study period. Of these, 70 patients (who delivered) tested positive for SARs-CoV-2 during their pregnancy and 210 patients who were asymptomatic (prior to universal testing) or had a negative SARs-CoV-2 test were matched to the index cases. Of the 70 patients who tested positive for SARs-CoV-2, $22(32.4 \%)$ were diagnosed with a HDP. Of the 210 patients with negative tests or who were not tested but asymptomatic, 38 (13.3\%) were diagnosed with HDP (Figure S1).

Baseline demographics were significantly different for race and social history. Patients who tested positive for COVID-19 were more likely to be Black/African American and have a history of recreational drug use during pregnancy (Table 1). Patients who tested positive for COVID-19 also delivered at a significantly earlier mean gestational age than patients who tested negative, $38 \mathrm{w} 0 \mathrm{~d} \pm 2.4 \mathrm{vs}$. $38 \mathrm{w} 7 \mathrm{~d} \pm 1.8$, respectively $(p=0.05)$. Gravidity, parity, number of prior vaginal and Cesarean sections, medical comorbidities, and alcohol use and/or tobacco use, did not significantly differ between groups. Overall, patients who reported a previous pregnancy complication were more likely to have a negative COVID-19 test. Except for HDP, there were no significant differences in pregnancy complications between index cases and controls (Table S1). 
Table 1. Maternal Characteristics and Demographics of Study Population

Characteristic

\begin{tabular}{|c|c|c|c|c|c|}
\hline & $n$ & Mean & $n$ & Mean & $p$ \\
\hline Age & 210 & $29.7 \pm 6.0$ & 70 & $28.7 \pm 4.9$ & 0.13 \\
\hline Race & & & & & 0.01 \\
\hline White & $93(80.9)$ & & $22(19.1)$ & & \\
\hline Black & $77(65.8)$ & & $40(34.2)$ & & \\
\hline All other & $40(83.3)$ & & $8(16.7)$ & & \\
\hline \multicolumn{6}{|l|}{ Medical Comorbidities } \\
\hline Chronic Hypertension & $9(4.3)$ & & $7(10.0)$ & & 0.74 \\
\hline Diabetes Mellitus & $4(1.9)$ & & $4(5.7)$ & & 0.10 \\
\hline Asthma & $24(11.5)$ & & $10(14.3)$ & & 0.54 \\
\hline Cardiovascular Disease & $3(1.4)$ & & $0(0)$ & & - \\
\hline COPD & $1(0.5)$ & & $1(1.4)$ & & 0.41 \\
\hline VTE & $1(0.5)$ & & $1(1.4)$ & & 0.41 \\
\hline Other & $41(19.5)$ & & $11(15.7)$ & & 0.48 \\
\hline
\end{tabular}

Social Habits

\begin{tabular}{|c|c|c|c|c|c|}
\hline Alcohol & $1(0.5)$ & & $0(0)$ & & - \\
\hline Tobacco & $4(1.9)$ & & $1(1.4)$ & & 0.80 \\
\hline Recreational Drugs & $6(2.9)$ & & $6(8.6)$ & & 0.04 \\
\hline BMI & 203 & $33.2 \pm 7.0$ & 70 & $32.6 \pm 5.3$ & 0.46 \\
\hline Gravidity & 210 & $2.8 \pm 1.8$ & 70 & $2.8 \pm 2.0$ & 0.99 \\
\hline Parity & 210 & $1.1 \pm 1.2$ & 70 & $1.06 \pm 1.4$ & 0.98 \\
\hline No. Prior SVD & 210 & $0.8 \pm 1.2$ & 70 & $0.8 \pm 1.3$ & 0.74 \\
\hline No. Prior CS & 210 & $0.3 \pm 0.7$ & 70 & $0.2 \pm 0.5$ & 0.70 \\
\hline Gestational Age Delivery (wk) & 210 & $38.7 \pm 1.8$ & 70 & $38.0 \pm 2.4$ & 0.05 \\
\hline
\end{tabular}

Data are Mean \pm SD or $n(\%)$, unless otherwise specified.

Bold indicates that data are significantly different 
Patients with COVID-19 diagnosed within their pregnancy were significantly more likely to be diagnosed with a hypertensive disorder of pregnancy $(p<0.01$; Table 2$)$. Overall rates of gestational hypertension $(5.7 \%$ vs $3.8 \%, p=0.500)$ and preeclampsia without severe features $(7.1 \%$ vs $2.4 \%, p=0.061)$ were not statistically significant, while preeclampsia with severe features $(18.6 \%$ vs $7.1 \%, p=0.006)$ was significantly higher in patients with a known COVID-19 diagnosis in pregnancy (Table S2). One patient had HELLP syndrome and 1 patient had eclampsia. Both were COVID-19 negative. The effect of SARsCoV-2 infection on overall rate of HDP was modified by BMI, parity, preeclampsia in a prior pregnancy, comorbid chronic hypertension, asthma, prior cesarean section, recreational drug use during pregnancy, and race (Table S3). Laboratory values and inflammatory markers were not included in the analysis secondary to lack of a standard laboratory order set across institutions. In adjusted analyses (Table 3), hypertensive disorders of pregnancy were associated with greater BMI (odds ratio [aOR] 1.1, 95\% confidence interval $[\mathrm{Cl}] 1.04-1.16, p=0.01)$, COVID-19 diagnosis ( $\mathrm{aOR}=3.68, \mathrm{Cl} 1.67-8.10, \mathrm{p}=0.01$ ), asthma (OR 2.69, $\mathrm{Cl} 1.07-6.74, \mathrm{p}=0.04$ ), chronic hypertension (OR 7.54, $\mathrm{Cl} 2.27-25.08$ ), and preeclampsia in previous pregnancy $(\mathrm{OR} 9.98, \mathrm{Cl} 2.72-36.64, \mathrm{p}=0.01)$. A history of Cesarean section was associated with a decreased risk of having a HDP (OR 0.009, 0.02-0.56) (Fig. 1).

\begin{tabular}{|c|c|c|c|c|}
\hline & & \multicolumn{2}{|c|}{ Hypertensive Disorder of Pregnancy. } & \multirow[b]{2}{*}{$p$} \\
\hline & & No & Yes & \\
\hline COVID-19 Status & Total & $\mathrm{n}(\%)$ & n (\%) & \multirow[t]{3}{*}{0.01} \\
\hline Negative & 210 & $182(86.7)$ & $28(13.3)$ & \\
\hline Positive & 70 & $48(68.6)$ & $22(31.4)$ & \\
\hline
\end{tabular}

\begin{tabular}{|c|c|c|c|}
\hline Predictor & $\begin{array}{l}\text { Odds } \\
\text { Ratio }\end{array}$ & $\begin{array}{l}\text { Confidence Interval } \\
(95 \%)\end{array}$ & $p$ \\
\hline BMI & 1.10 & $1.04-1.16$ & 0.01 \\
\hline COVID-19 Positive & 3.68 & $1.67-8.10$ & 0.01 \\
\hline Asthma Diagnosis & 2.69 & $1.07-6.74$ & 0.04 \\
\hline Chronic Hypertension Diagnosis & 7.54 & $2.27-25.08$ & 0.01 \\
\hline History of Prior Cesarean Section & 0.09 & $0.02-0.56$ & 0.01 \\
\hline $\begin{array}{l}\text { Preeclampsia as Previous Pregnancy } \\
\text { Complication }\end{array}$ & 9.98 & $2.72-36.64$ & 0.01 \\
\hline
\end{tabular}


When cases were stratified by diagnosis of COVID-19 and severity of COVID-19 symptomology, HDP were more likely to manifest in asymptomatic positive patients (Figure S2). HDP was diagnosed in $17.1 \%$ of asymptomatic COVID-19 patients, $5.7 \%$ of patients with mild symptoms, $7.1 \%$ of patients with moderate symptoms, and $1.4 \%$ of patients requiring intensive care. However, our study was not powered for this subgroup analysis. When COVID-19 positive cases were subdivided by gestational age at time of positive SARs-CoV-2 test (prior to 32 weeks' gestation [early] or after 32 weeks' gestation[late]), HDP was associated with early (OR $=13.00, \mathrm{Cl} 3.67-46.04 ; \mathrm{p}<0.001)$ but not late (OR 1.92, $\mathrm{Cl} 0.92-4.0 ; \mathrm{p}=0.082)$ SARs-CoV-2 infection when compared to negative controls. Of COVID-19 positive patients, early COVID-19 conferred a higher risk of HDP $(\mathrm{OR}=6.29, \mathrm{Cl} 1.64-24.07 ; \mathrm{p}=0.007)$ (Figure S3).

Patients with a positive SARs-CoV-2 test in pregnancy most commonly presented with fever $(22.9 \%)$, dyspnea (17.1\%), headache (15.7\%), and cough (15.7\%) (Table S4). The majority of patients were asymptomatic or were managed as outpatients (68.6\%). Eighteen of the 70 positive COVID-19 patients (25.7\%) had mild-moderate disease and were managed as inpatients (either for delivery or symptom management), and 4 patients (5.7\%) required intensive care. Pregnancy and fetal outcomes did not differ between groups (Tables S5 and S6). Patients diagnosed with COVID-19 had significantly lower absolute lymphocyte counts $(1.97 \pm 0.7$ vs $1.5 \pm 0.7, \mathrm{p}<0.001)$ and significantly higher C-reactive protein $(C R P)$ $(25.3$, interquartile range [IQR] $11.3,64.3$ vs 12.2 , IQR $6.2,19.3)$, creatinine $(0.72 \pm 0.26$ vs $0.63 \pm 0.14)$, and uric acid ( $5.9 \pm 1.4$ vs. $4.6 \pm 1.2)$. Leukocytes $(p=0.11)$, thrombocytes $(p=0.46)$, protein-to-creatinine ratio $(p=0.66)$, transaminases (alanine aminotransferase [ALT], $p=0.93$; aspartate aminotransferase [AST], $p$ $=0.51)$, lactate dehydrogenase $(\mathrm{LDH})(p=0.12)$, and vitamin $\mathrm{D}(\mathrm{p}=0.26)$ did not statistically differ between groups (Table S7).

\section{Discussion}

We found that SARs-CoV-2 infection in pregnancy is associated with significantly higher incidence of HDP. After controlling for other variables, patients with SARs-CoV-2 infections were nearly 4 times more likely to have a diagnosis of HDP compared to patients without a history of SARs-CoV-2 infection in pregnancy. SARs-CoV-2 infection was the third highest contributor to HDP diagnoses, only behind previous history of preeclampsia and comorbid chronic hypertension (Fig. 1). Overall, $31.4 \%$ of patients with a COVID-19 during their pregnancy developed a HDP, compared to $13.3 \%$ of controls. Our results are consistent with the available literature ${ }^{18,24}$. While one meta-analysis ${ }^{9}$ did not demonstrate this association, the studies included had heterogenous designs, different primary outcomes, and most lacked a control group. The one study within the meta-analysis with matched controls found a higher incidence of preeclampsia in pregnant patients with COVID-19 (7.7\% vs. 4.3\%, OR 1.84, Cl 1.00-3.36) [23].

Rosenbloom and Raghuraman (2021) found that a COVID-19 diagnosis conferred an approximate 2 -fold risk of HDP (hazard ratio (HD) 1.93; Cl 1.13-3.31) [24]. This was especially true if COVID-19 was diagnosed prior to 32 weeks' gestation, with the HR of 2.17 ( $\mathrm{Cl} 1.11-4.24)$. This is consistent with our findings. Madden et al (2021) found overall rates of gestational hypertension of $9.0 \%$ and $3.6 \%(p<$ $0.001)$, preeclampsia without severe features of $3.6 \%$ and $1.4 \%(p=0.034)$, and preeclampsia with severe 
features $5.4 \%$ and $3.6 \%(p=0.12)$ for patients with and without COVID-19, respectively [25]. They did not report an aggregate risk, however, and they did not control for other variables.

Metz et al. (2021) found that for patients with COVID-19, HDP was the most common indication among preterm births. In contrast to our study, they found a relative risk of 1.61 ( $95 \% \mathrm{Cl} 1.18-2.20)$ for severe COVID-19, with rates of HDP increasing with the severity of COVID-19 manifestation [22]. However, the impact of confounders was not ascertained. In our study, patients with a positive SARs-CoV-2 test and those who were asymptomatic had the highest likelihood of developing HDP. This is an important finding as patients with positive screening test may be at a higher risk of developing HDP, even if asymptomatic. While COVID-19 conferred a significant increased likelihood of having a diagnosis of HDP in our study, a diagnosis of chronic hypertension and having preeclampsia in a previous pregnancy conferred the greatest risk for HDP. However, COVID-19 remained a significant risk factor for HDP after controlling for these confounders.

Patients with COVID-19 were more likely to have lower absolute lymphocyte count and elevated CRP and uric acid. There was a trend toward a higher ferritin level. However, it should be noted that laboratory values were not analyzed as a predictor of HDP in our study given the relatively small proportion of control patients with inflammatory markers and the lack of a standard COVID-19 laboratory order-set across institutions. However, these results are consistent with the inflammatory state associated with COVID-19. Inflammation has been shown to play a significant role in the pathogenesis of HDP [26-28]. Furthermore, HDP has been associated with alterations in proinflammatory cytokines [26-27, 29]. The inflammatory response is a central clinical characteristic of SARs-CoV infection, with well-established extrapulmonary systemic hyperinflammation secondary to a proinflammatory state driven by the release of host cytokines [30-34]. Thus, it is likely that the acute inflammatory state, even if subclinical, may provoke or exacerbate HDP.

It has been reported that COVID-19 modulates angiotensin-converting enzyme 2 (ACE-2) within the placenta, which may provide one component in the pathogenesis of HDP in pregnancies complicated by SARs-CoV-2 infection [35]. ACE-2 has been shown to be heavily expressed in the placenta, including cells involved in decidualization, trophoblast invasion, vascular remodeling, and placental blood flow [36]. ACE2 is upregulated in pregnancy, and alterations in the levels of angiotensin II (vasoconstrictor) and angiotensin-(1-7) (vasodilator) caused by SARs-CoV-2 can lead to vasoconstriction, inflammation and pro-coagulopathic effects seen in $\mathrm{HDP}^{20}$. Furthermore, Mendoza et al (2020) showed abnormalities in angiogenic factors related to COVID-19 infection that resulted in increased risk of a preeclampsia-like syndrome [37]. Finally, it has been shown that placentas of women infected with SARs-CoV-2 have higher rates of decidual arteriopathy when compared to controls [38]. Together, these reports provide compelling evidence for the pathogenesis behind the observed increased risk of HDP in pregnant patients with COVID-19.

The timing of SARs-CoV-2 infection appears to be important. Early infection (before 32 weeks' gestation) was associated with a significantly increased risk of HDP compared to negative controls. Infections 
occurring after 32 weeks' gestation showed a trend toward increased risk of HDP, however was not statistically significant. Our study was not powered for this outcome and it is likely that a larger sample would have generated a significant increased risk for late SARs-CoV-2 infection compared to COVID-19 negative controls. When comparing only COVID-19 positive pregnancies, early infection conferred a significantly higher risk for HDP than late infection. This observation is consistent with the inflammatory explanation of increased HDP risk in COVID-19. Through modulation of angiogenic factors and inflammatory cytokines, it is likely that COVID-19 exerts maximal impact on placental physiology at earlier gestations, enabling these physiologic changes to manifest as HDP over time.

Our study has several strengths. Most studies to date that have reported increased risk of HDP associated with COVID-19 have lacked a control group. Our study incorporated a control group of unaffected patients. We compared COVID-19 cases to 3 controls that delivered in temporal proximity to the index case, thus eliminating inherent temporal biases as a result of fluctuations in the prevalence of COVID-19 cases. Furthermore, a large control group allowed us to conduct detailed statistical analyses to elucidate an association between SARs-CoV-2 infections and HDP. Other studies to date have lacked a larger control group.

The main limitation of this study is the retrospective design. With any retrospective study, there is potential misclassification based on nonreported or incorrectly recorded ICD-10 codes.

Additionally, given the scarcity of SARs-CoV-2 diagnostic tests early in the study period, approximately one-third of patients did not have a diagnostic test. This increases the potential for misclassification (e.g. including an asymptomatic COVID-19 positive case in the control group). However, we believe that this would potentially decrease the strength of association of COVID-19 and HDP, therefore the association found in this study may be an underestimate. There is also the possibility that patients could have tested positive at an outside institution during their pregnancy but did not disclose their positive test.

Additionally, our study was underpowered for analysis of important subcategories, such as the effects of inflammatory markers on HDP within COVID-19 positive cases. It is recognized that there are overlapping signs (e.g. transaminitis) and symptoms (e.g headache) associated with COVID-19 and HDP. Accordingly, clinicians should take these overlapping features into account when making clinical decisions regarding HDP in COVID-19 positive pregnant patients.

\section{Conclusion}

The results from our study suggest that pregnant patients with a history of COVID-19 disease in pregnancy are at increased risk for developing a HDP, regardless of symptomatology. HDP has been reported to account for a significant increase in iatrogenic preterm birth in patients with COVID-1922. Clinicians should be aware of this association and closely monitor patients with a history of SARs-CoV-2 infection for potential complications associated with HDP. Collectively, we should aim to develop strategies to mitigate iatrogenic complications related HDP in pregnant patients with COVID-19. 


\section{Declarations}

Funding: None

Conflicts of Interests/Competing interests: None

Availability of data and material: Upon Request

Code availability: Not applicable

Authors' contributions: M Baracy: Protocol/project development; Data Collection; Data analysis; Manuscript writing/editing. F Afzal: Data Collection; Manuscript writing/editing. SM Szpunar: Protocol/project development; Data analysis; Manuscript writing/editing. M Tremp: Data Collection. K Grace: Data Collection. M Liovas: Data Collection. MF Aslam: Protocol/project development; Manuscript writing/editing

Ethics approval: This study was approved by the Ascension St. John and Ascension Providence Institutional Review Boards (IRB) on 06/12/2020 (Reference \#1617953).

Consent to participate: Not applicable

Consent for publication: Not applicable

\section{Acknowledgements}

This study is dedicated to the late Dr. David M. Zalenski who was integral to the COVID-19 response as the OB/GYN department chair at Ascension St. John Hospital. In addition to practicing obstetrics and gynecology, Dr. Zalenski was an educator and leader for the St. John OB/Gyn residency program. His kind presence is sorely missed.

\section{References}

1. Zhu, N. et al. China Novel Coronavirus Investigating and Research Team. A Novel Coronavirus from Patients with Pneumonia in China, 2019. N Engl J Med, 20 (8), 727-733 https://doi.org/10.1056/NEJMoa2001017 (2020 Feb).

2. Holshue, M. L. et al. N Engl J Med, 5 (10), 929-936 https://doi.org/10.1056/NEJMoa2001191 (2020 Mar). for the Washington State 2019-nCoV Case Investigation Team. First Case of 2019 Novel Coronavirus in the

3. World Health Organization. WHO coronavirus disease (COVID-19) dashboard. Accessed March 29th, 2021. https://covid19.who.int/

4. Hansen, J. N., Hine, J. \& Strout, T. D. COVID-19 and preeclampsia with severe features at 34-weeks gestation. Am J Emerg Med. 2021 Jan;39:252.e3-252.e5. doi: 10.1016/j.ajem.2020.06.052. Epub 
2020 Jun 25. PMID: 33008706; PMCID: PMC7315969.

5. Garcia Rodriguez, A. et al. SARS-COV-2 infection during pregnancy, a risk factor for eclampsia or neurological manifestations of COVID-19? Case report.BMC Pregnancy Childbirth. 2020 Oct6;20(1):587. doi: 10.1186/s12884-020-03275-2. PMID: 33023500; PMCID: PMC7538036

6. Azarkish, F. et al. Preeclampsia and the crucial postpartum period for Covid-19 infected mothers: A case report. Pregnancy Hypertens, 23, 136-139 https://doi.org/10.1016/j.preghy.2020.10.012 (2021 Mar).

7. Di Mascio, D. et al. Outcome of coronavirus spectrum infections (SARS, MERS, COVID-19) during pregnancy: a systematic review and meta-analysis. Am J Obstet Gynecol MFM, 2 (2), 100107 https://doi.org/10.1016/j.ajogmf.2020.100107 (2020 May).

8. Stumpfe, F. M. et al. SARS-CoV-2 Infection in Pregnancy - a Review of the Current Literature and Possible Impact on Maternal and Neonatal Outcome. Geburtshilfe Frauenheilkd, 80 (4), 380-390 https://doi.org/10.1055/a-1134-5951 (2020 Apr).

9. Huntley, B. J. F. et al. Adverse Pregnancy Outcomes Among Individuals With and Without Severe Acute Respiratory Syndrome Coronavirus 2 (SARS-CoV-2): A Systematic Review and Meta-analysis. Obstet Gynecol. 2021 Apr 1;137(4):585-596. doi: 10.1097/AOG.0000000000004320. PMID: 33706357; PMCID: PMC7984633.

10. Fassett, M. J. et al. Universal SARS-Cov-2 Screening in Women Admitted for Delivery in a Large Managed Care Organization. Am J Perinatol, 37 (11), 1110-1114 https://doi.org/10.1055/s-00401714060 (2020 Sep).

11. Huntley, B. J. F. et al. Rates of Maternal and Perinatal Mortality and Vertical Transmission in Pregnancies Complicated by Severe Acute Respiratory Syndrome Coronavirus 2 (SARS-Co-V-2) Infection: A Systematic Review. Obstet Gynecol. 2020 Aug;136(2):303-312. doi:

10.1097/AOG.0000000000004010. PMID: 32516273.

12. Allotey, J. et al. Clinical manifestations, risk factors, and maternal and perinatal outcomes of coronavirus disease 2019 in pregnancy: living systematic review and meta-analysis BMJ 2020 Sep 1; 370:m3320doi:10.1136/bmj.m3320

13. WAPM (World Association of Perinatal Medicine) Working Group on COVID-19. Maternal and perinatal outcomes of pregnant women with SARS-CoV-2 infection. Ultrasound Obstet Gynecol. 2020 Sep 14;57(2):232-241. doi: 10.1002/uog.23107. Epub 2021 Jan 21. PMID: 32926494.

14. Yang, R. et al. Pregnant women with COVID-19 and risk of adverse birth outcomes and maternal-fetal vertical transmission: a population-based cohort study in Wuhan, China. BMC Med, 19 (1), 330 https://doi.org/10.1186/s12916-020-01798-1 (2020 Oct).

15. Di Mascio, D. et al. Risk factors associated with adverse fetal outcomes in pregnancies affected by Coronavirus disease 2019 (COVID-19): a secondary analysis of the WAPM study on COVID-19. J Perinat Med. 2020 Nov 26;48(9):950-958. doi: 10.1515/jpm-2020-0355. PMID: 32975205.

16. Adhikari, E. H. et al. Pregnancy Outcomes Among Women With and Without Severe Acute Respiratory Syndrome Coronavirus 2 Infection. JAMA Netw Open. 2020 Nov 2;3(11):e2029256. doi: 
10.1001/jamanetworkopen.2020.29256. PMID: 33211113; PMCID: PMC7677755.

17. Brandt, J. S. et al. Epidemiology of coronavirus disease 2019 in pregnancy: risk factors and associations with adverse maternal and neonatal outcomes. Am J Obstet Gynecol. 2021 Apr;224(4):389.e1-389.e9. doi: 10.1016/j.ajog.2020.09.043. Epub 2020 Sep 25. PMID: 32986989; PMCID: PMC7518835.

18. Jering, K. S. et al. Clinical Characteristics and Outcomes of Hospitalized Women Giving Birth With and Without COVID-19. JAMA Intern Med. Published online January, 15, https://doi.org/10.1001/jamainternmed.2020.9241 (2021).

19. Wei, S. Q., Bilodeau-Bertrand, M., Liu, S. \& Auger, N. The impact of COVID-19 on pregnancy outcomes: a systematic review and meta-analysis. CMAJ. 2021 Mar 19:cmaj.202604. doi:

10.1503/cmaj.202604. Epub ahead of print. PMID: 33741725

20. Narang, K. et al. SARS-CoV-2 Infection and COVID-19 During Pregnancy: A Multidisciplinary Review. Mayo Clin Proc. 2020 Aug;95(8):1750-1765. doi: 10.1016/j.mayocp.2020.05.011. Epub 2020 May 30. PMID: 32753148; PMCID: PMC7260486.

21. Coronado-Arroyo, J. C., Concepción-Zavaleta, M. J., Zavaleta-Gutiérrez, F. E. \& Concepción-Urteaga, L. A. Is COVID-19 a risk factor for severe preeclampsia? Hospital experience in a developing country. Eur J Obstet Gynecol Reprod Biol, 256, 502-503 https://doi.org/10.1016/j.ejogrb.2020.09.020 (2021 Jan).

22. Metz, T. D. et al. Eunice Kennedy Shriver National Institute of Child Health and Human Development (NICHD) Maternal-Fetal Medicine Units (MFMU) Network. Disease Severity and Perinatal Outcomes of Pregnant Patients With Coronavirus Disease 2019 (COVID-19). Obstet Gynecol. 2021 Apr 1;137(4):571-580. doi: 10.1097/AOG.0000000000004339. PMID: 33560778; PMCID: PMC7984765.

23. Ahlberg, M. et al. Association of SARS-CoV-2 Test Status and Pregnancy Outcomes. JAMA, 324 (17), 1782-1785 https://doi.org/10.1001/jama.2020.19124 (2020).

24. Rosenbloom, J. I., Raghuraman, N., Carter, E. B. \& Kelly, J. C. Coronavirus disease 2019 infection and hypertensive disorders of pregnancy. Am J Obstet Gynecol. 2021 Mar 3:S0002-9378(21)00150-2. doi: 10.1016/j.ajog.2021.03.001. Epub ahead of print. PMID: 33675794; PMCID: PMC7926183.

25. Madden, N. et al. 32 COVID-19 and new hypertensive disease in pregnancy. Am J Obstet Gynecol, 224 (2), S23-4 Epub 2021 Feb 1. PMCID: PMC7848673. (2021 Feb).

26. Tali, Y. S., Lyimo, M. A., Luzzatto, L. \& Massawe, S. N. Hypertensive disorders of pregnancy are associated with an inflammatory state: evidence from hematological findings and cytokine levels. BMC Pregnancy Childbirth. 2019 Jul 9;19(1):237. doi: 10.1186/s12884-019-2383-7. PMID: 31288789; PMCID: PMC6617701.

27. Matthiesen, L. et al. Immunology of preeclampsia. Chem Immunol Allergy. 2005;89:49-61. doi: 10.1159/000087912. PMID: 16129952.

28. Redman, C. W. \& Sargent, I. L. Immunology of pre-eclampsia. Am J Reprod Immunol, 63 (6), 534-543 https://doi.org/10.1111/j.1600-0897.2010.00831.x (2010 Jun). 
29. Lockwood, C. J. et al. Preeclampsia-related inflammatory cytokines regulate interleukin- 6 expression in human decidual cells. Am J Pathol, 172 (6), 1571-1579 https://doi.org/10.2353/ajpath.2008.070629 (2008 Jun).

30. Qin, C. et al. Dysregulation of Immune Response in Patients With Coronavirus 2019 (COVID-19) in Wuhan, China.Clin Infect Dis. 2020 Jul28;71(15):762-768. doi: 10.1093/cid/ciaa248. PMID: 32161940; PMCID: PMC7108125

31. Wu, C. et al. Risk Factors Associated With Acute Respiratory Distress Syndrome and Death in Patients With Coronavirus Disease 2019 Pneumonia in Wuhan, China. JAMA Intern Med. 2020 Jul 1;180(7):934-943. doi: 10.1001/jamainternmed.2020.0994. Erratum in: JAMA Intern Med. 2020 Jul 1;180(7):1031. PMID: 32167524; PMCID: PMC7070509.

32. Azkur, A. K. et al. Immune response to SARS-CoV-2 and mechanisms of immunopathological changes in COVID-19. Allergy. 2020 Jul;75(7):1564-1581. doi: 10.1111/all.14364. PMID: 32396996; PMCID: PMC7272948

33. Siddiqi, H. K. \& Mehra, M. R. COVID-19 illness in native and immunosuppressed states: A clinicaltherapeutic staging proposal. J Heart Lung Transplant, 39 (5), 405-407 https://doi.org/10.1016/j.healun.2020.03.012 (2020 May).

34. Maxwell, A. J. et al. Identification of key signaling pathways induced by SARS-CoV2 that underlie thrombosis and vascular injury in COVID-19 patients.J Leukoc Biol. 2021 Jan;109(1):35-47. doi: 10.1002/JLB.4COVR0920-552RR. Epub 2020 Nov 26. PMID: 33242368; PMCID: PMC7753679

35. Jing, Y. et al. Potential influence of COVID-19/ACE2 on the female reproductive system. Mol Hum Reprod. 2020 Jun 1;26(6):367-373. doi: 10.1093/molehr/gaaa030. PMID: 32365180; PMCID: PMC7239105.

36. Valdes, G. et al. Utero-placental expression of angiotensin-(1-7) and ACE2 in the pregnant guineapig. Reproductive Biology and Endocrinology, 11 (5), 1-8 (2013).

37. Mendoza, M. et al. Pre-eclampsia-like syndrome induced by severe COVID-19: a prospective observational study. BJOG, 127 (11), 1374-1380 https://doi.org/10.1111/1471-0528.16339 (2020 Oct).

38. Shanes, E. D. et al. Placental Pathology in COVID-19. Am J Clin Pathol. 2020 Jun 8;154(1):23-32. doi: 10.1093/ajcp/aqaa089. PMID: 32441303; PMCID: PMC7279066.

\section{Figures}




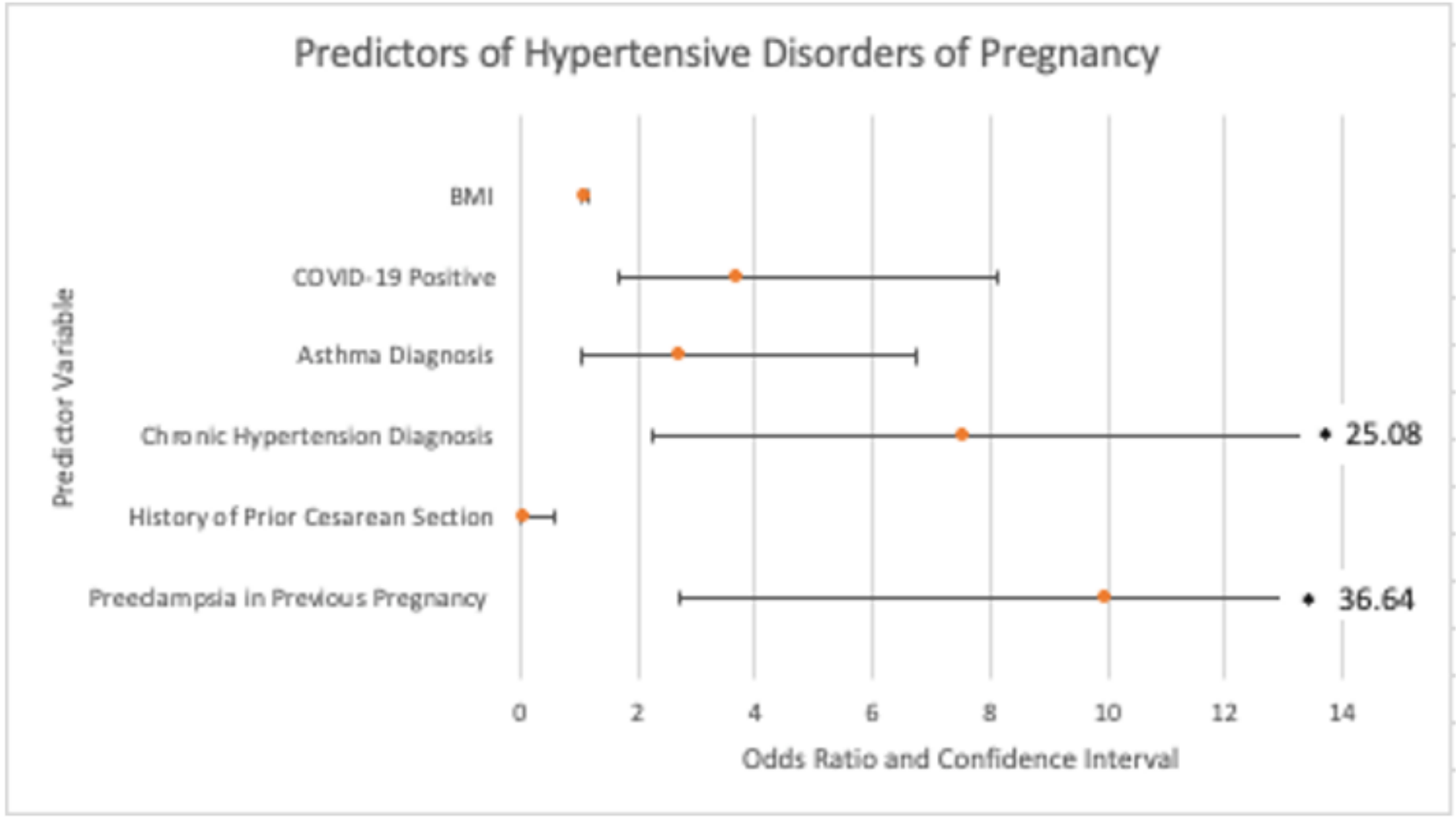

\section{Figure 1}

Adjusted Odds Ratio for Hypertensive Disorder of Pregnancy by COVID-19 and other Risk Factors

\section{Supplementary Files}

This is a list of supplementary files associated with this preprint. Click to download.

- SupplementaryTablesandFigures.docx 